\title{
VARIETIES OF COMPLETELY REGULAR SEMIGROUPS
}

\author{
NORMAN R. REILLY
}

(Received 12 September 1983)

Communicated by T. E. Hall

\begin{abstract}
If $\mathscr{C} \mathscr{S}$ (respectively, $\mathcal{O}$ ) denotes the class of all completely simple semigroups (respectively, semigroups that are orthodox unions of groups) then $\mathscr{C} \mathscr{S}$ (respectively, $\mathcal{O}$ ) is a variety of algebras with respect to the operations of multiplication and inversion. The main result shows that the lattice of subvarieties of $\mathscr{C} \mathscr{S} \vee \mathcal{O}$ is a precisely determined subdirect product of the lattice of subvarieties of $\mathscr{C} \mathscr{S}$ and the lattice of subvarieties of $\mathcal{O}$. A basis of identities is obtained for any variety $\mathscr{V} \subset \mathscr{E} \mathscr{S} \vee \mathcal{O}$ in terms of bases of identities for $\mathscr{V} \cap \mathscr{C} \mathscr{S}$ and $\mathscr{V} \cap \mathcal{O}$. Several operators on the lattice of subvarieties of $\mathscr{L} \mathscr{S} \vee \mathcal{O}$ are also introduced and studied.
\end{abstract}

1980 Mathematics subject classification (Amer. Math. Soc.): 20 M 07, 20 M 10.

\section{Introduction}

A semigroup is said to be completely regular if it is a union of groups. The class $\mathscr{C} \mathscr{R}$ of all completely regular semigroups, considered as algebras with the binary operation of multiplication and the unary operation of inversion within each subgroup, is a variety determined by the identities

$$
x(y z)=(x y) z, \quad x=x x^{-1} x, \quad\left(x^{-1}\right)^{-1}=x, \quad x x^{-1}=x^{-1} x .
$$

Considerable progress has been made in recent years in the investigation of various sublattices of the lattice $\mathscr{L}(\mathscr{C} \mathscr{R})$ of all subvarieties of $\mathscr{C} \mathscr{R}$. The most complete answer has been obtained for the lattice of subvarieties $\mathscr{L}(\mathscr{B})$ of the variety $\mathscr{B}$ of all bands (that is, semigroups of idempotents). This lattice is completely known on account of the work of Birjukov [1], Fennemore [2] and

(c) 1985 Australian Mathematical Society $0263-6115 / 85 \$ A 2.00+0.00$ 
Gerhard [3]. The lattice $\mathscr{L}(\mathscr{G})$ of all subvarieties of the variety $\mathscr{G}$ of all groups is, of course, part of the picture and, though extensively studied, is not and cannot be expected to ever be completely determined. Pressing on from the lattice of all varieties of groups, Petrich [9] determined the lattice of subvarieties of the variety of orthodox bands of groups as the direct product of $\mathscr{L}(\mathscr{G})$ and $\mathscr{L}(\mathscr{B})$. (Recall that $S$ is orthodox if $e, f \in S, e^{2}=e$ and $f^{2}=f$ imply that $(e f)^{2}=e f$ and that $S$ is a band of groups if $S$ is completely regular and $\mathscr{H}$ is a congruence.) Gerhard and Petrich [4] have completely determined (modulo the lattice of varieties of groups) the lattice of varieties of orthodox completely regular semigroups for which the subsemigroup of idempotents is regular (axya = axaya $)$.

The lattice $\mathscr{L}(\mathscr{C} \mathscr{S})$ of all subvarieties of the variety $\mathscr{C} \mathscr{S}$ of all completely simple semigroups turns out to be surprisingly complicated, but much is now known about this lattice; see for example Jones [8], Petrich and Reilly [15], [16], [17] and [18] and Rasin [19].

A completely regular semigroup $S$ is said to be pseudo-orthodox if eSe is orthodox, for all $e^{2}=e \in S$.

The variety of all pseudo-orthodox completely regular semigroups is denoted by $\mathscr{P O}$ and the variety of all pseudo-orthodox bands of groups is denoted by $\mathscr{P O O \mathscr { G }}$. Recently, Hall and Jones [6] showed that $\mathscr{B} \mathscr{S} \vee \mathscr{B}=\mathscr{P O O \mathscr { B }}$. Independently, Hall and Jones [6] and Rasin [20] have shown that the lattice $\mathscr{L}(\mathscr{C} \mathscr{S} \vee \mathscr{B})$ of all subvarieties of $\mathscr{C} \mathscr{S} \vee \mathscr{B}$ is a subdirect product of $\mathscr{L}(\mathscr{C} \mathscr{S})$ and $\mathscr{L}(\mathscr{B})$.

If we let $\mathcal{O}$ denote the class of all orthodox completely regular semigroups and let $I$ denote the class of all completely regular semigroups $S$ such that $a, b \in S$ and $p$ in the subsemigroup of $S$ generated by the idempotents implies that $a p b \mathscr{H} a p p^{-1} b$, then Hall and Jones [6] also establishes that

$$
\mathscr{C} \mathscr{S} \vee \mathcal{O}=I \cap \mathscr{P O}
$$

where $\mathscr{P O}$ is the variety of all pseudo-orthodox completely regular semigroups.

In the main result of this paper, it is shown that the lattice of subvarieties of $\mathscr{C} \mathscr{S} \vee \mathcal{O}$ is a (precisely determined) subdirect product of $\mathscr{L}(\mathscr{C} \mathscr{S})$ and $\mathscr{L}(\mathcal{O})$. It is further shown that every element $\mathscr{V}$ of this lattice is the intersection of certain varieties related to $\mathscr{V} \cap \mathscr{C} \mathscr{S}$ and $\mathscr{V} \cap \mathcal{O}$ together with $\mathscr{P O O}$. From this it is possible to obtain a basis of identities for $\mathscr{V}$ in terms of bases for $\mathscr{V} \cap \mathscr{C} \mathscr{S}$ and $\mathscr{V} \cap \mathcal{O}$.

In Section 6 , the operator $\mathscr{P}$ is considered in some detail. For instance, its behaviour on $\mathscr{L}(\mathscr{B})$ is completely determined and it is shown to be an endomorphism of $\mathscr{L}(\mathscr{P} O \mathscr{B} \mathscr{G})$.

One operator on classes of completely regular semigroups that plays an important role thoroughout is defined on any such class $\mathscr{K}$ by $(\mathscr{K})^{+}=\{S \in \mathscr{C} \mathscr{R}$ : there is a congruence $\rho$ on $S$ such that $\rho \subseteq \mathscr{H}$ and $S / \rho \in \mathscr{K}\}$. It is shown, for instance, that $I=\mathcal{O}^{+}$. Another operator is defined on each class $\mathscr{K}$ by $(\mathscr{K})^{p}=$ $\{S \in \mathscr{C} \mathscr{R}$ : there is a congruence $\rho$ on $S$ such that $\rho \cap \mathscr{H}=\iota$ and $S / \rho \in \mathscr{K}\}$. Various relationships between these operators are considered in Section 7. 


\section{Background}

We adopt the notation and terminology of Howie [7], to which the reader is referred for basic information. However, we will use the term completely regular to describe any semigroup which is a union of groups. The following result due to Clifford is the cornerstone of the whole theory.

THEOREM 2.1 [7]. If $S$ is a completely regular semigroup then $S$ is a semilattice of completely simple semigroups: that is, $S=\bigcup\left\{S_{i}: i \in I\right\}$ where I is a semilattice, the $S_{i}$ are disjoint completely simple semigroups and $S_{i} S_{j} \subseteq S_{i j}$.

We will refer to the subsemigroups $S_{i}$ of $S$ in Theorem 2.1 as the completely simple components of $S$.

The following notation will be convenient.

$\mathscr{T}-$ the variety of one element semigroups.

$\mathscr{S} \ell-$ the variety of semilattices.

$\mathscr{N} \mathscr{B}-$ the variety of normal bands.

$\mathscr{B}$ - the variety of bands.

$\mathscr{G}$ - the variety of groups.

$\mathscr{S} \ell \mathscr{G}$ - the variety of semilattices of groups.

$\mathscr{C} \mathscr{S}$ - the variety of completely simple semigroups.

$\mathcal{O}$ - the variety of orthodox completely regular semigroups.

$\mathscr{B} \mathscr{G}$ - the variety of bands of groups ( $\equiv$ completely regular and $\mathscr{H}$ a congruence).

$\mathscr{U} \mathscr{B} \mathscr{G}$ - the variety of those bands of groups such that $S / \mathscr{H} \in \mathscr{U} \in \mathscr{L}(\mathscr{B})$.

$\mathscr{N} \mathscr{B} \mathscr{G}$ - the variety of normal bands of groups.

$O \mathscr{B G}$ - the variety of orthodox bands of groups.

$\mathcal{O N} \mathscr{B} \mathscr{G}$ - the variety of orthodox normal bands of groups.

$\mathscr{C} \mathscr{R}$ - the variety of completely regular semigroups.

$\mathscr{L}(\mathscr{V})$ - the variety of all subvarieties of the variety $\mathscr{V}$.

$\mathscr{E}(\mathscr{S})$ - the set of idempotents of the semigroup $S$.

$\langle E(S)\rangle$ - the subsemigroup of $S$ generated by $E(S)$.

$\left[u_{\alpha}=v_{\alpha}: \alpha \in A\right]-$ the variety of all completely regular semigroups satisfying the identities $u_{\alpha}=v_{\alpha}(\alpha \in A)$.

$x^{0}=x x^{-1}$, for any $x \in S \in \mathscr{C} \mathscr{R}$.

Particularly noteworthy among the relationships between the above varieties is the following. 
LEMMA $2.2[11] . \mathscr{N} \mathscr{B} \mathscr{G}=\mathscr{C} \mathscr{S} \vee \mathscr{S} \ell$.

A basis of identities for most of the varieties listed above can be found in Petrich [12]. We assume implicitly that every general identity involves $n$ variables. When we wish to emphasize this we will write $u\left(x_{1}, \ldots, x_{n}\right)=v\left(x_{1}, \ldots, x_{n}\right)$ although it should be noted that this does not necessarily imply that all variables appear on both sides.

The term variety will always mean variety of completely regular semigroups and the identities (1) are assumed to hold throughout.

The following "inverse of a product" law for completely regular semigroups will be useful.

LEMMA 2.3 [14]. For any elements $a, b$ of a completely regular semigroup, $(a b)^{-1}=(a b)^{0} b^{-1}(b a)^{0} a^{-1}(a b)^{0}$.

It is well known [7] that if $\theta$ is an equivalence relation on a semigroup $S$ then there is a maximum congruence $\rho$ on $S$ contained in $\theta$. We denote by $\mu_{S}$ the maximum congruence contained in Green's relation $\mathscr{H}$.

A congruence $\rho$ on $S$ is said to be idempotent pure if and only if $e^{2}=e$ and $(e, a) \in \rho$ implies that $a^{2}=a$. Clearly a congruence $\rho$ on a completely regular semigroup is idempotent pure if and only if $\rho \cap \mathscr{H}=\imath$. Also, if $\rho_{i}(i \in I)$ are idempotent pure congruences, then so is $\mathrm{V}\left\{\rho_{i}: i \in I\right\}$.

Since every completely regular semigroup is regular (in the sense that $a \in a S a$, for all $a \in S$ ) we have

LEMMA 2.4 [7]. Let $\rho$ be a congruence on $S \in \mathscr{C} \mathscr{R}$ and $a \in S$ be such that $a \rho \in E(S / \rho)$. Then $a \rho=e \rho$, for some $e \in E(S)$.

If $\rho$ is a congruence on a subsemigroup $T$ of a semigroup $S$, then we will denote by $\rho^{\#}$ the smallest congruence on $S$ which contains $\rho$.

Lemma 2.5 (Hall and Jones [6]). Let $S \in \mathscr{C} \mathscr{R}, e \in E(S)$ and $\rho$ be a congruence on eSe. Then

(i) $\left.\rho^{\#}\right|_{e s e}=\rho$,

(ii) if $\rho$ is idempotent pure, then so is $\rho^{\#}$,

(iii) if $\rho_{e}$ is an idempotent pure congruence on eSe for each $e \in E(S)$, then $\rho=\bigvee\left\{\rho_{e}^{\#}: e \in E(S)\right\}$ is an idempotent pure congruence on $S$.

If $\mathscr{U}$ is a variety and $S \in \mathscr{C} \mathscr{S}$, then the minimum $\mathscr{U}$-congruence on $S$ is the smallest congruence $\rho$ on $S$ such that $S / \rho \in \mathscr{U}$. 
One of the important features of orthodox semigroups is that for any orthodox semigroup $S$ there is an idempotent pure congruence $\rho$ on $S$ such that the idempotents in $S / \rho$ commute (see [7], ch. VI). If $S$ is completely regular, then $S / \rho$ is completely regular with commuting idempotents, and so is a semilattice of groups. Thus we have

LEMMA 2.6. For any $S \in \mathcal{O}$, the minimum $\mathscr{S} \ell \mathscr{G}$-congruence is idempotent pure.

Since orthodox semigroups are central to our discussions, we will need some further basic facts about them.

LEMMA 2.7 [7]. Let $S$ be a completely regular semigroup. Then $S$ is orthodox if and only if every completely simple component of $S$ is orthodox.

LEMMA 2.8 [7]. Let $S$ be an orthodox semigroup, $a \in S$ and $a^{\prime}$ be an inverse of $a$. Then $a^{\prime} E(S) a \subseteq E(S)$.

LEMMA 2.9. Let $S$ be a completely simple semigroup. Define a sequence of subsets of $S$ inductively as follows:

$$
E_{1}=E(S), \quad E_{n+1}= \begin{cases}E_{n} E_{n} & \text { if } n+1 \text { is even, } \\ \left\{x^{-1} y x: x \in S, y \in E_{n}\right\} & \text { if } n+1 \text { is odd } .\end{cases}
$$

Let $N=\bigcup\left\{E_{n}: n=1,2,3, \ldots\right\}$. Define the relation $\rho$ on $S$ by

$$
(a, b) \in \rho \Leftrightarrow a^{0}=b^{0} \text { and } a b^{-1} \in N .
$$

Then $\rho$ is the smallest congruence on $S$ such that $S / \rho$ is orthodox.

Proof. If $e \in E_{n} \cap E(S)$, then $e=e e=e^{-1} e e \in E_{n+1}$ so that $E(S) \subseteq E_{n}$, for all $n$. Also, if $x \in E_{n}$ then $x=x^{0} x=\left(x^{0}\right)^{-1} x x^{0} \in E_{n+1}$ so that $E_{n} \subseteq E_{m}$, for $n \leqslant m$. Hence $N$ is clearly a subsemigroup of $S$. A simple induction argument using Lemma 2.3, will show that $N$ is closed under inversion $\left(x \rightarrow x^{-1}\right)$ while, by construction, $N$ is closed under "conjugation" $\left(y \rightarrow x^{-1} y x\right)$. For each $e \in E(S)$, let $N_{e}=H_{e} \cap N$. Clearly $N_{e}$ is a normal subgroup of $H_{e}$. From this it is clear that $\rho$ is an equivalence relation on $S$. Now let $(a, b) \in \rho$ and $x$ be any element of $S$. Let $a=n b, n \in N_{e}, e=a^{0}=b^{0}$. Then $a x \mathscr{H} b x, n(b x)^{0} \mathscr{H} a x, n(b x)^{0} \in N$ and $a x=n b x=\left[n(b x)^{0}\right] b x$, so that $(a x, b x) \in \rho$. Also

$$
\begin{aligned}
x a & =x n b=x n^{0} n b \\
& =\left(x n^{0}\right) n\left(x n^{0}\right)^{0} b \\
& =\left(x n^{0}\right) n\left(x n^{0}\right)^{-1}\left(x n^{0}\right) b \\
& =\left(x n^{0}\right) n\left(x n^{0}\right)^{-1} x b \quad\left(\text { since } n^{0}=b^{0}\right)
\end{aligned}
$$


where $x a \mathscr{H} x b,\left(x n^{0}\right) n\left(x n^{0}\right)^{-1} \mathscr{H} x n^{0} \mathscr{H} x b$ and $\left(x n^{0}\right) n\left(x n^{0}\right)^{-1} \in N$. Hence $(x a, x b) \in \rho$ and $\rho$ is a congruence.

Now, for any $e, f \in E(S)$, ef $\in N$ so that $(e \rho)(f \rho)=(e f) \rho=(e f)^{0} \rho \in$ $E(S / \rho)$. Thus, by Lemma $2.4, S / \rho$ is orthodox. On the other hand, let $\sigma$ be any congruence on $S$ such that $S / \sigma$ is orthodox. A simple induction argument together with Lemma 2.8 shows that for all $n \in N,\left(n, n^{0}\right) \in \sigma$. Hence for $(a, b) \in \rho$, say $a=n b$ where $n \in N \cap H_{e}$ and $e=a^{0}=b^{0}, a=n b \sigma n^{0} b=b$. Thus $\rho \subseteq \sigma$, as required.

Definition 2.10 . Let $\mathscr{X}$ be any property of completely regular semigroups (respectively, class of completely regular semigroups). Then a completely regular semigroup $S$ is said to be pseudo- $\mathscr{X}$ if $e S e$ has the property $\mathscr{K}$ (respectively, $e S e \in \mathscr{X}$ ) for all $e \in E(S)$. The class of all pseudo- $\mathscr{K}$ completely regular semigroups will be denoted by $\mathscr{P} \mathscr{X}$.

Although this concept had appeared earlier in the context of pseudo-varieties of finite languages and automata, its importance in the context of varieties of completely regular semigroups was established by Hall and Jones [6]. We gather here some basic facts about $\mathscr{P}$ and we study $\mathscr{P}$ in greater depth in Section 6.

The following observations are elementary:

$$
\mathscr{P} \mathscr{T}=\mathscr{R} \mathscr{B}, \quad \mathscr{P} \mathscr{S} \ell=\mathscr{N} \mathscr{B}, \quad \mathscr{P} \mathscr{B}=\mathscr{B}, \quad \mathscr{P}(\mathscr{G})=\mathscr{P}(\mathscr{C} \mathscr{S})=\mathscr{C} \mathscr{P}
$$

while, for any $\mathscr{U}, \mathscr{V} \in \mathscr{L}(\mathscr{C} \mathscr{S})$,

$$
\mathscr{P} \mathscr{P}(\mathscr{U})=\mathscr{P}(\mathscr{U}), \mathscr{P}(\mathscr{U} \cap \mathscr{V})=\mathscr{P}(\mathscr{U}) \cap \mathscr{P}(\mathscr{V}) .
$$

More surprisingly, we have

$$
\text { LEMMA } 2.11[6] . \mathscr{P} \mathscr{B} \mathscr{G}=\mathscr{B} \mathscr{G} \text {. }
$$

Thus $(\mathscr{P O}) \mathscr{B} \mathscr{G}=\mathscr{P}(\mathscr{O B G})$ and we may write simply $\mathscr{P} O \mathscr{B} \mathscr{G}$ for this variety.

Notation 2.12 . For any variety $\mathscr{U}$ of completely simple semigroups, we write

$$
\overline{\mathscr{U}}=\{S \in \mathscr{C} \mathscr{S}: \text { all subgroups of } S \text { lie in } \mathscr{U}\} \text {. }
$$

Clearly $\overline{\mathscr{U}}=\overline{\mathscr{U} \cap \mathscr{G}}$ and it follows from Petrich and Reilly [15] that $\overline{\mathscr{U}}$ is a variety of completely simple semigroups. It is easy to see from this that $\mathscr{P} \mathscr{U}=\overline{\mathscr{U}}$, for all $\mathscr{U} \in \mathscr{L}(\mathscr{C} \mathscr{S})$. Now, for any $\mathscr{U}, \mathscr{V} \in \mathscr{L}(\mathscr{C} \mathscr{S}), \overline{\mathscr{U}} \vee \overline{\mathscr{V}}=\overline{\mathscr{U} \vee \mathscr{V}}$ so that

$$
\mathscr{P}(\mathscr{U} \vee \mathscr{V})=\overline{\mathscr{U} \vee \mathscr{V}}=\overline{\mathscr{U}} \vee \overline{\mathscr{V}}=\mathscr{P} \mathscr{U} \vee \mathscr{P} \mathscr{V} .
$$

Combining this observation with the fact that $\mathscr{P}(\mathscr{C} \mathscr{S})=\mathscr{C} \mathscr{S}$, we have

LEMMA 2.13. $\mathscr{P}$ induces a retraction on $\mathscr{L}(\mathscr{C} \mathscr{S})$. 
Finally,

LEMMA 2.14 [12]. If $\mathscr{V} \in \mathscr{L}(\mathscr{C} \mathscr{R})$ and $\mathscr{V}=\left[u_{\alpha}=v_{\alpha}: \alpha \in A\right]$, then

$$
\begin{aligned}
\mathscr{P} \mathscr{V} & =\left[u_{\alpha}\left(x^{0} x_{1} x^{0}, \ldots, x^{0} x_{n} x^{0}\right)=v_{\alpha}\left(x^{0} x_{1} x^{0}, \ldots, x^{0} x_{n} x^{0}\right): \alpha \in A\right] \\
& =\left[u_{\alpha}\left(x x_{1} x, \ldots, x x_{n} x\right)=v_{\alpha}\left(x x_{1} x, \ldots, x x_{n} x\right): \alpha \in A\right] .
\end{aligned}
$$

In particular, since $\mathcal{O}=\left[a^{0} b^{0}=\left(a^{0} b^{0}\right)^{0}\right]$,

$$
\mathscr{P O}=\left[(x a x)^{0}(x b x)^{0}=\left((x a x)^{0}(x b x)^{0}\right)^{0}\right] .
$$

\section{3. $\mathscr{H}$-coextensions}

In this section we associate with each $\mathscr{V} \in \mathscr{L}(\mathscr{C} \mathscr{R})$ a new variety $\mathscr{V}^{+}$and consider various properties associated with $\mathscr{V}^{+}$.

Notation 3.1. For any $\mathscr{V} \in \mathscr{L}(\mathscr{C} \mathscr{R})$, let $\mathscr{V}^{+}=\{S \in \mathscr{C} \mathscr{R}: S / \mu \in \mathscr{V}\}$.

Clearly $S \in \mathscr{V}^{+}$if and only if there exists some idempotent separating congruence $\rho$ on $S$ such that $S / \rho \in \mathscr{V}$. For the special case where $\mathscr{V}$ is a variety of bands, the variety $\mathscr{V}^{+}$was introduced by Rasin [20].

\section{LEMMA 3.2. For any $\mathscr{V} \in \mathscr{L}(\mathscr{C} \mathscr{R}), \mathscr{V}^{+} \in \mathscr{L}(\mathscr{C} \mathscr{R})$.}

Proof. Clearly $\mathscr{V}^{+}$is closed under products and subobjects. Let $S \in \mathscr{V}^{+}, \rho$ be a congruence on $S$ and $T=S / \rho$. Now $\left(\rho \vee \mu_{S}\right) / \rho \subseteq \mathscr{H}_{T}$ while $T /\left[\left(\rho \vee \mu_{S}\right) / \rho\right]$ $\cong S /\left(\rho \vee \mu_{S}\right)$. Since $S /\left(\rho \vee \mu_{S}\right)$ is a homomorphic image of $S / \mu_{S} \in \mathscr{V}$, it follows that $T /\left[\left(\rho \vee \mu_{S}\right) / \rho\right] \in \mathscr{V}$ so that $T \in \mathscr{V}^{+}$.

Some obvious examples of $\mathscr{V}^{+}$are $\mathscr{G}=\mathscr{T}^{+}, \mathscr{S} \ell \mathscr{G}=\mathscr{S} \ell^{+}, \mathscr{N} \mathscr{B} \mathscr{G}=\mathscr{N} \mathscr{B}$, $\mathscr{C} \mathscr{S}=\mathscr{R} \mathscr{B}^{+}$and $\mathscr{B} \mathscr{G}=\mathscr{B}^{+}=\mathscr{B} \mathscr{G}^{+}$. In fact, for any $\mathscr{U} \in \mathscr{L}(\mathscr{B})$, we have $\mathscr{U} \mathscr{B} \mathscr{G}=$ $\mathscr{U}^{+}$. A less obvious example is provided in Proposition 3.5 below.

The operator $(-)^{+}$also determines certain modular sublattices of $\mathscr{L}(\mathscr{C} \mathscr{R})$.

LeMma 3.3. For any $\mathscr{V} \in \mathscr{L}(\mathscr{C} \mathscr{R})$, the interval $\left[\mathscr{V}, \mathscr{V}^{+}\right]$is modular.

Proof. Let $F$ be the free completely regular semigroup on a countably infinite set of generators and, for any $\mathscr{U} \in \mathscr{L}(\mathscr{C} \mathscr{R})$ let $\rho_{\mathscr{U}}$ denote the fully invariant congruence on $F$ corresponding to the variety $\mathscr{U}$. Let $F_{\mathscr{Q}}=F / \rho_{\mathscr{Q}}$.

Let $\mathscr{V} \subseteq \mathscr{U} \subseteq \mathscr{V}^{+}$. Then there exists a congruence $\rho \subseteq \mathscr{H}$ on $F_{\mathscr{Y}^{+}}$such that $F_{\mathscr{V}^{+}} / \rho \in \mathscr{V} \subseteq \mathscr{U}$. Hence $\rho_{\mathscr{Q} /} / \rho_{\mathscr{V}^{+}} \subseteq \mathscr{H}$ so that there is an anti-isomorphism of the 
interval $\left[\mathscr{V}, \mathscr{V}^{+}\right]$onto a sublattice of the lattice $L$ of those congruences on $F_{\mathscr{V}^{+}}$ that are contained in $\mathscr{H}$. Since $L$ is modular ([7], Ex. II.4.3), so also is [ $\mathscr{V}, \mathscr{V}^{+}$].

Let $I$ denote the class of all completely regular semigroups $S$ such that

$$
a p b \mathscr{H} a p^{0} b \quad \text { for all } p \in\left\langle E_{S}\right\rangle, a, b \in S \text {. }
$$

THEOREM 3.4 [5]. The class $I$ is a variety of completely regular semigroups and $I \cap \mathscr{P O}=\mathscr{C} \mathscr{S} \vee \mathcal{O}$.

Proposition 3.5. $I=\mathscr{O}^{+}=\left\{S \in \mathscr{C} \mathscr{R}:\left\langle E_{S}\right\rangle \in \mathscr{B} \mathscr{G}\right\}$.

Proof. Let $A$ denote the third class in the statement of the proposition. That $I \subseteq \mathcal{O}^{+}$is part of Lemma 5.2 of [6]. Let $S \in \mathcal{O}^{+}$and $\rho$ be a congruence on $S$ such that $\rho \subseteq \mathscr{H}$ and $S / \rho \in \mathcal{O}$. Let $\operatorname{ker} \rho=\left\{a \in S: a \rho a^{0}\right\}$. Since $S / \rho$ is orthodox, $\operatorname{ker} \rho$ is a subsemigroup of $S$. Let $\sigma$ be the restriction of $\rho$ to $\operatorname{ker} \rho$. Then $\sigma \subseteq \mathscr{H}$ and $(\operatorname{ker} \rho) / \sigma$ is a band. Hence $\operatorname{ker} \rho$ is a band of groups and since $\left\langle E_{S}\right\rangle \subseteq \operatorname{ker} \rho$, it follows that $\left\langle E_{S}\right\rangle$ is a band of groups and $\mathcal{O}^{+} \subseteq A$.

Now let $S \in A$. Since $\left\langle E_{S}\right\rangle \in \mathscr{B} \mathscr{G}$, it follows that

$$
a^{0} p b^{0} \mathscr{H} a^{0} p^{0} b^{0} \quad \text { for all } p \in\left\langle E_{S}\right\rangle, a, b \in S .
$$

Hence, for any $p \in\left\langle E_{S}\right\rangle, a, b \in S$,

$$
\begin{aligned}
& a p b=a\left(a^{0} p b^{0}\right) b \\
& =(a e)\left(a^{0} p b^{0}\right)(e b) \quad \text { where } e=\left(a^{0} p b^{0}\right)^{0} \\
& \mathscr{H} \quad(a e)\left(a^{0} p^{0} b^{0}\right)(e b) \quad \text { by }(4) \text { and since all bracketed } \\
& \text { elements are } \mathscr{J} \text {-equivalent } \\
& =a p^{0} b \\
& \text { since } e=\left(a^{0} p^{0} b^{0}\right)^{0} \text { by (4) }
\end{aligned}
$$

as required.

Corollary 3.6. (i) $\mathcal{O}^{+} \cap \mathscr{P O}=\mathcal{O} \vee \mathscr{C} \mathscr{S}$.

(ii) The interval $[\mathcal{O}, I]$ is modular.

Proof. This is immediate from Lemma 3.3, Theorem 3.4 and Proposition 3.5.

Our next objective is to obtain a basis of identities for $\mathscr{V}^{+}$in terms of any basis for $\mathscr{V}$. Recall that we are assuming throughout the identities (1) so that we are only listing those identities required in addition to (1). We require some preliminary observations. 
LEMMA 3.7 [7]. Let $\rho_{0}$ be a reflexive symmetric relation on a semigroup $S$. Let $\rho$ consist of all pairs $(a, b)$ such that there exist $s_{i}, t_{i}, p_{i}, q_{i} \in S^{1}$ with $\left(p_{i}, q_{i}\right) \in \rho_{0}$ $(i=1, \ldots, n)$ and

$$
\begin{aligned}
a & =s_{1} p_{1} t_{1} \\
s_{1} q_{1} t_{1} & =s_{2} p_{2} t_{2} \\
s_{2} q_{2} t_{2} & =s_{3} p_{3} t_{3} \\
& \vdots \\
s_{n} q_{n} t_{n} & =b
\end{aligned}
$$

Then $\rho$ is the congruence on $S$ generated by $\rho_{0}$.

LEMMA 3.8. Let $\mathscr{V}=\left[u_{\alpha}=v_{\alpha}: \alpha \in A\right] \in \mathscr{L}(\mathscr{C} \mathscr{R})$ and $S \in \mathscr{C} \mathscr{R}$. Let $\rho_{0}$ be the set of all pairs $(p, q) \in S \times S$ such that for some $a_{1}, \ldots, a_{n} \in S, \alpha \in A$ either

(i) $p=q$ or (ii) $p=u_{\alpha}\left(a_{1}, \ldots, a_{n}\right)$ and $q=v_{\alpha}\left(a_{1}, \ldots, a_{n}\right)$ or (iii) $p=$ $v_{\alpha}\left(a_{1}, \ldots, a_{n}\right)$ and $q=u_{\alpha}\left(a_{1}, \ldots, a_{n}\right)$.

Then $\rho$, as defined in Lemma 3.7, is the smallest congruence on $S$ such that $S / \rho \in \mathscr{V}$.

THEOREM 3.9. Let $\mathscr{V}=\left[u_{\alpha}=v_{\alpha}: \alpha \in A\right] \in \mathscr{L}(\mathscr{C} \mathscr{R})$. Then

$$
\mathscr{V}^{+}=\left[u_{\alpha}^{0}=v_{\alpha}^{0},\left(x u_{\alpha} y\right)^{0}=\left(x v_{\alpha} y\right)^{0}: \alpha \in A\right] .
$$

Proof. Let $\mathscr{W}$ denote the right-hand side of (6) and let $S \in \mathscr{V}^{+}$. Then there exists a congruence $\rho$ on $S$ such that $\rho \subseteq \mathscr{H}$ and $S / \rho \in \mathscr{V}$. Therefore, for any $a, a_{1}, \ldots, a_{n}, b \in S$,

$$
(a \rho) u_{\alpha}\left(a_{1} \rho, \ldots, a_{n} \rho\right)(b \rho)=(a \rho) v_{\alpha}\left(a_{1} \rho, \ldots, a_{n} \rho\right)(b \rho)
$$

so that

$$
\left(a u_{\alpha}\left(a_{1}, \ldots, a_{n}\right) b, a v_{\alpha}\left(a_{1}, \ldots, a_{n}\right) b\right) \in \rho \subseteq \mathscr{H} .
$$

Hence $S$ satisfies $\left(x u_{\alpha} y\right)^{0}=\left(x v_{\alpha} y\right)^{0}$ and, similarly, $u_{\alpha}^{0}=v_{\alpha}^{0}$. Thus $\mathscr{V}^{+} \subseteq \mathscr{W}$. Now let $S \in \mathscr{W}$ and let $\rho_{0}$ and $\rho$ be defined as in Lemma 3.8, so that $S / \rho \in \mathscr{V}$. Let $(a, b) \in \rho$ and $s_{i}, t_{i}, p_{i}, q_{i} \in S$, with $\left(p_{i}, q_{i}\right) \in \rho_{0}$, satisfy (5). Since $S^{\prime} \in \mathscr{W}$, $s_{i} p_{i} t_{i} \mathscr{H}_{s_{i}} q_{i} t_{i}$, for all $i$. Hence $a \mathscr{H} b, \rho \subseteq \mathscr{H}$ and $S \in \mathscr{V}^{+}$. Thus $\mathscr{V}^{+}=\mathscr{W}$, as required.

Theorem 3.9 can be used to obtain bases of identities for the examples of $\mathscr{V}^{+}$ given after Lemma 3.2, but these are all well known. However, in [6], $I=\mathcal{O}^{+}$was shown to be a variety by exhibiting an infinite basis of identities satisfied precisely by the members of $I$. Using Theorem 3.9, a much simpler basis can be obtained as follows. 
COROLlary 3.10. $I=\left[\left(x a^{0} b^{0} y\right)^{0}=\left(x\left(a^{0} b^{0}\right)^{0} y\right)^{0}\right]$.

Proof. Since, by Proposition 3.5, $I=\mathcal{O}^{+}$, it follows from Theorem 3.9 and from $\mathcal{O}=\left[a^{0} b^{0}=\left(a^{0} b^{0}\right)^{0}\right]$ that

$$
\begin{aligned}
I & =\left[\left(a^{0} b^{0}\right)^{0}=\left(\left(a^{0} b^{0}\right)^{0}\right)^{0},\left(x a^{0} b^{0} y\right)^{0}=\left(x\left(a^{0} b^{0}\right)^{0} y\right)^{0}\right] \\
& =\left[\left(x a^{0} b^{0} y\right)^{0}=\left(x\left(a^{0} b^{0}\right)^{0} y\right)^{0}\right]
\end{aligned}
$$

since the first identity is trivial.

$$
\text { 4. } \mathscr{L}\left(\mathcal{O}^{+} \cap \mathscr{P O}\right)=\mathscr{L}(\mathcal{O} \vee \mathscr{C} \mathscr{S})
$$

Hall and Jones [6] showed that $\mathscr{P O O B} \mathscr{G}=\mathscr{C} \mathscr{S} \vee \mathscr{B}$, while Rasin [20] and Hall and Jones [6] independently have completely determined the lattice $\mathscr{L}(\mathscr{C} \mathscr{S} \vee \mathscr{B})$ $(=\mathscr{L}(\mathscr{P O O B G})$ ) as a subdirect product of $\mathscr{L}(\mathscr{C} \mathscr{S})$ and $\mathscr{L}(\mathscr{B})$. In this section we extend this result by showing that $\mathscr{L}\left(\mathcal{O}^{+} \cap \mathscr{P O}\right)$ is a subdirect product of $\mathscr{L}(\mathscr{C} \mathscr{S})$ and $\mathscr{L}(\mathcal{O})$.

As a first step we show that the lattice $\mathscr{L}\left(\mathcal{O}^{+} \cap \mathscr{P O}\right)$ is generated by the sublattices $\mathscr{L}(\mathscr{C} \mathscr{S})$ and $\mathscr{L}(\mathcal{O})$.

The first observation is drawn from [6].

Proposition 4.1. (i) For any $\mathscr{V} \in \mathscr{L}\left(\mathcal{O}^{+} \cap \mathscr{P O}\right), \mathscr{V}=(\mathscr{V} \cap \mathscr{C} \mathscr{P}) \vee(\mathscr{V} \cap \mathcal{O})$ (ii) for any $\mathscr{V} \in \mathscr{L}(\mathscr{P} O \mathscr{B} \mathscr{G}), \mathscr{V}=(\mathscr{V} \cap \mathscr{C} \mathscr{S}) \vee(\mathscr{V} \cap \mathscr{B})$.

Proof. (i) Let $\mathscr{V} \in \mathscr{L}\left[\mathcal{O}^{+} \cap \mathscr{P} \mathcal{O}\right]$. By Corollary 5.5 of [6], $\mathscr{V}=(\mathscr{V} \cap \mathcal{O}) \vee(\mathscr{V} \cap$ $\mathscr{N \mathscr { B }} \mathscr{G})$. But by Equation (6) of [11],

$$
\begin{aligned}
\mathscr{V} \cap \mathscr{N} \mathscr{B} \mathscr{G} & =((\mathscr{V} \cap \mathscr{N} \mathscr{B} \mathscr{G}) \cap \mathscr{S} \mathscr{L}) \vee((\mathscr{V} \cap \mathscr{N} \mathscr{B} \mathscr{G}) \cap \mathscr{C} \mathscr{S}) \\
& =(\mathscr{V} \cap \mathscr{S} \mathscr{L}) \vee(\mathscr{V} \cap \mathscr{C} \mathscr{S}) .
\end{aligned}
$$

Hence $\mathscr{V}=(\mathscr{V} \cap \mathcal{O}) \vee(\mathscr{V} \cap \mathscr{C} \mathscr{S})$.

Part (ii) is a part of [6], Corollary 5.7.

Next it will be shown that for every variety $\mathscr{M}$ of completely simple semigroups there is a largest variety of completely regular semigroups the completely simple members of which are precisely the members of $\mathscr{M}$. A basis of identities for this variety will be given in terms of any basis for $\mathscr{M}$.

Notation 4.2. For any variety $\mathscr{M}$ of completely simple semigroups, let $\mathscr{M}^{*}=\{S \in \mathscr{C} \mathscr{R}$ : every completely simple component of $S$ lies in $\mathscr{M}\}$.

It is clearly the case that for any $\mathscr{V} \in \mathscr{L}(\mathscr{C} \mathscr{R}), \mathscr{V} \subseteq(\mathscr{V} \cap \mathscr{C} \mathscr{P})^{*}$. 
Notation 4.3. For any identity $u=v$ in the variables $x_{1}, \ldots, x_{n}$ (so that each $x_{i}$ appears either in $u$ or $v$ or both) let $u^{*}=v^{*}$ denote the identity $u\left(x_{1}^{*}, \ldots, x_{n}^{*}\right)=$ $v\left(x_{1}^{*}, \ldots, x_{n}^{*}\right)$ where $x_{i}^{*}=e_{i} x_{i} e_{i}, e_{i}=\left(x_{i} x_{1} x_{2} \cdots x_{n} x_{i}\right)^{0}$. Note that all variables appear on both sides of $u^{*}=v^{*}$. For arbitrary elements $a_{i}(i=1, \ldots, n)$ of any completely regular semigroup, we give the notation $a_{i}^{*}$ the analogous interpretation.

THEOREM 4.4. Let $\mathscr{M}=\left[u_{\alpha}=v_{\alpha}: \alpha \in A\right]$ be a variety of completely simple semigroups.

(i) $\mathscr{M}^{*}$ is the largest variety of completely regular semigroups such that $\mathscr{H}^{*} \cap \mathscr{C} \mathscr{S}$ $=\mathscr{A}$.

(ii) $\mathscr{H}^{*}=\left[u_{\alpha}^{*}=v_{\alpha}^{*}: \alpha \in A\right]$.

Proof. It follows immediately from the definition of $\mathscr{H}^{*}$ that $\mathscr{H}^{*} \cap \mathscr{C} \mathscr{S}=\mathscr{M}$.

Let $\mathscr{W}$ denote the class described in (ii) and let $S \in \mathscr{M}^{*}$. For any $a_{i} \in S$ $(i=1, \ldots, n)$, the elements $a_{i}^{*}$ are in the same completely simple component of $S$. Hence, by hypothesis, $u_{\alpha}\left(a_{1}^{*}, \ldots, a_{n}^{*}\right)=v_{\alpha}\left(a_{1}^{*}, \ldots, a_{n}^{*}\right)$ or $u_{\alpha}^{*}\left(a_{1}, \ldots, a_{n}\right)=$ $v_{\alpha}^{*}\left(a_{1}, \ldots, a_{n}\right)$. Thus $S \in \mathscr{W}$ and $\mathscr{M}^{*} \subseteq \mathscr{W}$. Conversely, let $S \in \mathscr{W}$ and let $T$ be any completely simple component of $S$. Since $T$ is completely simple, for any $a_{1}, \ldots, a_{n} \in T,\left(a_{i} a_{1} \cdots a_{n} a_{i}\right)^{0}=a_{i}^{0}$ so that

$$
\begin{aligned}
u_{\alpha}\left(a_{1}, \ldots, a_{n}\right) & =u_{\alpha}\left(a_{1}^{*}, \ldots, a_{n}^{*}\right) \\
& =u_{\alpha}^{*}\left(a_{1}, \ldots, a_{n}\right) \\
& \left.=v_{\alpha}^{*}\left(a_{1}, \ldots, a_{n}\right) \quad \text { (since } S \in \mathscr{W}\right) \\
& =v_{\alpha}\left(a_{1}^{*}, \ldots, a_{n}^{*}\right) \\
& =v_{\alpha}\left(a_{1}, \ldots, a_{n}\right) .
\end{aligned}
$$

Hence $T \in \mathscr{M}$ and $S \in \mathscr{M}^{*}$. Thus $\mathscr{W} \subseteq \mathscr{M}^{*}$ and (ii) holds. In particular $\mathscr{M}^{*}$ is a variety of completely regular semigroups.

Finally, let $\mathscr{V}$ be a variety of completely regular semigroups such that $\mathscr{V} \cap \mathscr{C} \mathscr{S}=\mathscr{M}$. Then every completely simple component of $\mathscr{V}$ must lie in $\mathscr{M}$ so that $\mathscr{V} \subseteq \mathscr{M}^{*}$. Thus $\mathscr{K}^{*}$ is the largest such variety and the proof of the theorem is complete.

Next it will be shown that, modulo some restrictions, the join of any variety of completely simple semigroups with any variety of orthodox completely regular semigroups does not contain any new completely simple or orthodox semigroups. We will need the following useful result due to Jones [9] and Hall and Jones [6]:

LEMMA 4.5. The mappings $\mathscr{V} \rightarrow \mathscr{V} \cap \mathscr{C} \mathscr{S}, \mathscr{V} \rightarrow \mathscr{V} \cap \mathscr{G}$ and $\mathscr{V} \rightarrow \mathscr{V} \cap \mathscr{B}$ are retractions of $\mathscr{L}(\mathscr{C} \mathscr{R})$ onto $\mathscr{L}(\mathscr{C} \mathscr{S}), \mathscr{L}(\mathscr{G})$ and $\mathscr{L}(\mathscr{B})$, respectively. 
COROLlary 4.6. Let $\mathscr{U} \in \mathscr{L}(\mathscr{C} \mathscr{S})$ and $\mathscr{V} \in \mathscr{L}(\mathcal{O})$ be such that $\mathscr{V} \subseteq \mathscr{U}^{*}$. Then $(\mathscr{U} \vee \mathscr{V}) \cap \mathscr{C} \mathscr{S}=\mathscr{U}$.

Proof. By Lemma 4.5, $(\mathscr{U} \vee \mathscr{V}) \cap \mathscr{C} \mathscr{S}=(\mathscr{U} \cap \mathscr{C} \mathscr{S}) \vee(\mathscr{V} \cap \mathscr{C} \mathscr{S})=\mathscr{U} \vee(\mathscr{V}$ $\cap \mathscr{C} \mathscr{S})=\mathscr{U}$ since $\mathscr{V} \subseteq \mathscr{U}^{*}$.

Proposition 4.7. Let $\mathscr{U} \in \mathscr{L}(\mathscr{C} \mathscr{S})$ and $\mathscr{V} \in \mathscr{L}(\mathcal{O})$ be such that

$$
\mathscr{U} \cap \mathscr{G} \subseteq \mathscr{V} \cap \mathscr{G}, \mathscr{U} \cap \mathscr{B} \subseteq \mathscr{V} \cap \mathscr{B} \text {. }
$$

Then $(\mathscr{U} \vee \mathscr{V}) \cap \mathcal{O}=\mathscr{V}$.

Proof. Let $S \in(\mathscr{U} \vee \mathscr{V}) \cap \mathcal{O}$. Then $S$ is orthodox and for some $A \in \mathscr{U}$, $B \in \mathscr{V}$ and subdirect product $R \subseteq A \times B$ there is an epimorphism $\theta: R \rightarrow S$. Let the completely simple components of $B$ be $\left\{B_{i}: i \in I\right\}$. For each $i \in I$, let

$$
A_{i}=\left\{a \in A:(a, b) \in R, \text { for some } b \in B_{i}\right\} .
$$

Clearly $A_{i}$ is a completely simple subsemigroup of $A$, for all $i \in I$. Moreover, if $j \leqslant i(i, j \in I)$ then $A_{i} \subseteq A_{j}$. To see this, let $a \in A_{i}$. Then $x=(a, b) \in R$, for some $b \in B_{i}$. Let $y=(c, d) \in R$ where $d \in B_{j}$. Then $x(x y x)^{0}$ has second component in $B_{j}$ and first component equal to $a(a c a)^{0}=a a^{0}=a$. Thus $A_{i} \subseteq A_{j}$ as claimed.

So let $T$ be the strong semilattice (see [7]) of the completely simple semigroups $A_{i}$ where the connecting homomorphism $\theta_{i j}: A_{i} \rightarrow A_{j}$ for $j \leqslant i$, is the natural embedding.

Let $C=\left\{(t, b) \in T \times B\right.$ : for some $\left.i, t \in A_{i}, b \in B_{i}\right\}$.

Then $C$ is a subdirect product of $A$ and $B$ known as the spined product [7].

Let $R^{\prime}=\{(a, b) \in C:(a, b) \in R\}$. The only difference between $R^{\prime}$ and $R$ is that in $T$ (and $R^{\prime}$ ) we consider $A_{i}$ and $A_{j}$, for $i \neq j$, as disjoint, whereas in $A \times B$ they are both subsets of $A$. Thus it is easily seen that $R^{\prime}$ and $R$ are isomorphic.

From this it follows that $S$ is a homomorphic image of $R^{\prime}$ and therefore of $R^{\prime} / \tau$, where $\tau$ denotes the minimum congruence on $R^{\prime}$ such that the quotient is orthodox. Our goal therefore is to show that $R^{\prime} / \tau \in \mathscr{V}$.

We first construct a congruence on $T$. For each $i \in I$, let $\gamma_{i}$ denote the minimum 0 -congruence on $A_{i}$ (see Lemma 2.9). The $\gamma_{i}$ are compatible with the structural homomorphisms $\theta_{i j}$ in the sense that if $a, b \in A_{i}$ and $(a, b) \in \gamma_{i}$, then $\left(a \theta_{i j}, b \theta_{i j}\right) \in \gamma_{j}$. This follows immediately from Lemma 2.9. Hence $\gamma=$ $\cup\left\{\gamma_{i}: i \in I\right\}$ is a congruence on $T$.

Now let $\delta$ be the congruence on $C$ defined by $((a, b),(r, s)) \in \delta \Leftrightarrow(a, r) \in \gamma$ and $b=s$.

Next we claim that $\left.\delta\right|_{R^{\prime}}=\tau$. We postpone the details of the proof of this claim to Lemma 4.8 below and proceed with the remainder of the proof of the proposition. 
Now $T / \gamma$ is a strong semilattice of the completely simple semigroups $A_{i} / \gamma_{i}$ with structural homomorphisms $\varphi_{i j}$ induced by the $\theta_{i j}$ :

$$
(a \gamma) \varphi_{i j}=\left(a \theta_{i j}\right) \gamma \quad\left(a \in A_{i}\right) .
$$

Hence $C / \delta$ is the spined product $T / \gamma \times B$ and is a subdirect product of $T / \gamma$ and $B$.

As a strong semilattice of the $A_{i} / \gamma_{i}, T / \gamma$ is a subdirect product of semigroups of the form $\left(A_{i} / \gamma_{i}\right)^{0}$ with possibly one semigroup of the form $A_{i} / \gamma_{i}$. We assume that $B$, and therefore also $T$, is not completely simple. The case when $B$ is completely simple is similar but simpler. So, assuming that $B$ is not completely simple, we have that $\mathscr{S} \ell \subseteq \mathscr{V}$. Furthermore, any $A_{i} / \gamma_{i}$ (respectively, $\left.\left(A_{i} / \gamma_{i}\right)^{0}\right)$ is an orthodox completely simple semigroup (respectively, with zero) and so is of the form $G_{i} \times H_{i}$ (respectively, $\left(G_{i} \times H_{i}\right)^{0}$, where each $G_{i}$ is a group and each $H_{i}$ a band. Then for each $i$,

$$
\begin{aligned}
& G_{i} \in \mathscr{U} \cap \mathscr{G} \subseteq \mathscr{V} \cap \mathscr{G} \subseteq \mathscr{V}, \\
& H_{i} \in \mathscr{U} \cap \mathscr{B} \subseteq \mathscr{V} \cap \mathscr{B} \subseteq \mathscr{V}
\end{aligned}
$$

so that $A_{i} / \gamma_{i}$ and $\left(A_{i} / \gamma_{i}\right)^{0}$ lie in $\mathscr{V}$ for all $i \in I$. Hence $T / \gamma \in \mathscr{V}$. Since $B \in \mathscr{V}$, by assumption, it follows that $C / \delta \in \mathscr{V}$ so that $R^{\prime} / \tau \in \mathscr{V}$ and therefore $S \in \mathscr{V}$.

We now complete the proof of Proposition 4.7 with the following lemma.

LEMMA 4.8. $\left.\delta\right|_{R^{\prime}}=\tau$.

Proof. Since $A_{i} / \gamma_{i}$ is orthodox for all $i$, it follows from Lemma 2.7 that $T / \gamma$ is orthodox and so $R^{\prime} / \delta$ is orthodox.

Let us write $\delta^{\prime}=\left.\delta\right|_{R^{\prime}}$. Since $R^{\prime} / \delta^{\prime}$ is orthodox, $\tau \subseteq \delta^{\prime}$. Now $\delta \subseteq \mathscr{H}$ and so $\delta^{\prime} \subseteq \mathscr{H}$. Hence in order to establish that $\delta^{\prime} \subseteq \tau$ it suffices to show that

$$
w \in R^{\prime}, \quad\left(w, w^{0}\right) \in \delta^{\prime} \Rightarrow\left(w, w^{0}\right) \in \tau .
$$

Let $w=(r, s) \in R^{\prime}, r \in A_{i}, s \in B_{i}$ and $\left(w, w^{0}\right) \in \delta^{\prime}$. By the definition of $\delta$, we must have $s=s^{0}$ and $\left(r, r^{0}\right) \in \gamma_{i}$. Let $E_{n}$ be defined in $A_{i}$ as in Lemma 2.9. Then $\gamma_{i}$ is defined as in (2) and $r \in E_{n}$, for some $n$. We first show that

$$
\text { there exists } h \in E\left(B_{i}\right) \text { such that }(r, h) \in R^{\prime} \text { and }(r, h) \tau\left(r^{0}, h\right) \text {. }
$$

We proceed by induction on $n$. If $n=1$, then $r \in E\left(A_{i}\right)$ and $(r, s)=\left(r^{0}, s^{0}\right)$ so that (9) is satisfied. Now let (9) hold for all elements of $E_{n}$ and let $r \in E_{n+1}$.

Case (a), $n+1$ is even. Then $E_{n+1}=E_{n} E_{n}$ so that $r=a b$, for some $a, b \in E_{n}$. By the induction hypothesis, there exist $h, k \in E\left(B_{i}\right)$ such that $(a, h),(b, k) \in R^{\prime}$, 
$(a, h) \tau\left(a^{0}, h\right)$ and $(b, k) \tau\left(b^{0}, k\right)$. Therefore $h k \in E\left(B_{i}\right)$ since $B$ is orthodox and

$$
\begin{aligned}
(r, h k)= & (a b, h k) \\
= & (a, h)(b, k) \\
\tau & \left(a^{0}, h\right)\left(b^{0}, k\right) \\
\tau & {\left[\left(a^{0}, h\right)\left(b^{0}, k\right)\right]^{0} \quad \text { (since } R^{\prime} / \tau \text { is orthodox) } } \\
= & \left(\left(a^{0} b^{0}\right)^{0}, h k\right) \\
= & \left(r^{0}, h k\right) \quad \text { (since } A_{i} \text { is completely simple). }
\end{aligned}
$$

Thus (9) holds.

Case (b), $n+1$ is odd. Then, for some $a \in A_{i}, b \in E_{n}$, we must have $r=a^{-1} b a$. By the induction hypothesis, there is an element $k \in E\left(B_{i}\right)$ with $(b, k) \in R^{\prime}$ and $(b, k) \tau\left(b^{0}, k\right)$. Let $h \in B_{i}$ be such that $(a, h) \in R^{\prime}$. By Lemma $2.8, h^{-1} k h \in$ $E\left(B_{i}\right)$, since $B_{i}$ is orthodox. Then

$$
\begin{aligned}
\left(r, h^{-1} k h\right)= & \left(a^{-1} b a, h^{-1} k h\right) \\
= & (a, h)^{-1}(b, k)(a, h) \\
\tau & (a, h)^{-1}\left(b^{0}, k\right)(a, h) \\
\tau & {\left[(a, h)^{-1}\left(b^{0}, k\right)(a, h)\right]^{0} } \\
& \left(\text { by Lemma } 2.8, \text { since } R^{\prime} / \tau \text { is orthodox }\right) \\
= & \left(a^{-1} b^{0} a, h^{-1} k h\right)^{0} \\
= & \left(r^{0}, h^{-1} k h\right) \quad(\text { since } S \text { is completely simple }) .
\end{aligned}
$$

Thus (9) holds in this case also and so (9) holds in general. Now let $h \in E\left(B_{i}\right)$ be such that $(r, h) \tau\left(r^{0}, h\right)$. Then, since $B_{i}$ is orthodox and completely simple

$$
\begin{aligned}
w & =\left(r, s^{0}\right)=\left(r, s^{0} h s^{0}\right) \\
& =\left(r^{0}, s^{0}\right)(r, h)\left(r^{0}, s^{0}\right) \\
& \tau\left(r^{0}, s^{0}\right)\left(r^{0}, h\right)\left(r^{0}, s^{0}\right) \\
& =\left(r^{0}, s^{0}\right)=w^{0} .
\end{aligned}
$$

Thus (8) holds and the proof of Lemma 4.8 is complete.

THEOREM 4.9. The mapping

$$
\chi: \mathscr{V} \rightarrow(\mathscr{V} \cap \mathscr{C} \mathscr{S}, \mathscr{V} \cap \mathcal{O}) \quad \mathscr{V} \in \mathscr{L}\left(\mathcal{O}^{+} \cap \mathscr{P O}\right)
$$

is an isomorphism of $\mathscr{L}\left(\mathcal{O}^{+} \cap \mathscr{P O}\right)$ onto the subdirect product

$$
\begin{array}{r}
\mathscr{F}=\left\{(\mathscr{U}, \mathscr{V}) \in \mathscr{L}(\mathscr{C} \mathscr{S}) \times \mathscr{L}(\mathcal{O}): \text { (i) } \mathscr{V} \subseteq \mathscr{U}^{*}, \text { (ii) } \mathscr{U} \cap \mathscr{G}=\mathscr{V} \cap \mathscr{G}\right. \\
\text { and (iii) } \mathscr{U} \cap \mathscr{R} \mathscr{B} \subseteq \mathscr{V} \cap \mathscr{R} \mathscr{B}\}
\end{array}
$$


of $\mathscr{L}(\mathscr{C} \mathscr{S})$ and $\mathscr{L}(\mathcal{O})$. Moreover,

$$
\chi^{-1}:(\mathscr{U}, \mathscr{V}) \rightarrow \mathscr{U} \vee \mathscr{V} \quad((\mathscr{U}, \mathscr{V}) \in \mathscr{F})
$$

Proof. We first show that $\mathscr{F}$ is indeed a subdirect product. Let $\left(\mathscr{U}_{i}, \mathscr{V}_{i}\right) \in \mathscr{F}$, $i=1,2$. If $S \in \mathscr{V}_{1} \cap \mathscr{V}_{2} \cap \mathscr{C} \mathscr{S}$, then $S \in \mathscr{V}_{1} \cap \mathscr{C} \mathscr{S} \subseteq \mathscr{U}_{1}$ and similarly $S \in \mathscr{U}_{2}$ so that $\mathscr{V}_{1} \cap \mathscr{V}_{2} \cap \mathscr{C} \mathscr{S} \subseteq \mathscr{U}_{1} \cap \mathscr{U}_{2}$ and $\left(\mathscr{U}_{1}, \mathscr{V}_{1}\right) \wedge\left(\mathscr{U}_{2}, \mathscr{V}_{2}\right)$ satisfies condition (i). Conditions (ii) and (iii) are also easily verified so that $\left(\mathscr{U}_{1}, \mathscr{V}_{1}\right) \wedge\left(\mathscr{U}_{2}, \mathscr{V}_{2}\right) \in$ $\mathscr{F}$.

Now $\mathscr{V}_{i} \subseteq \mathscr{U}_{i}^{*}, i=1,2$, implies that $\mathscr{V}_{1} \vee \mathscr{V}_{2} \subseteq \mathscr{U}_{1}^{*} \vee \mathscr{U}_{2}^{*} \subseteq\left(\mathscr{U}_{1} \vee \mathscr{U}_{2}\right)^{*}$ so that $\left(\mathscr{U}_{1}, \mathscr{V}_{1}\right) \vee\left(\mathscr{U}_{2}, \mathscr{V}_{2}\right)$ satisfies (i). Condition (ii) is satisfied by Lemma 4.5 . For condition (iii), suppose that $\left(\mathscr{U}_{1} \vee \mathscr{U}_{2}\right) \cap \mathscr{R} \mathscr{B}=\mathscr{R} \mathscr{B}$. Then either there exists an $i \in\{1,2\}$ such that $\mathscr{R} \mathscr{B} \subseteq \mathscr{U}_{i}$ or else $\mathscr{L} \mathscr{Z} \subseteq \mathscr{U}_{i}$ and $\mathscr{R} \mathscr{Z} \subseteq \mathscr{U}_{j}$ where $\{i, j\}=$ $\{1,2\}$. In either case, it follows that $\mathscr{R} \mathscr{B} \subseteq\left(\mathscr{V}_{1} \vee \mathscr{V}_{2}\right) \cap \mathscr{R} \mathscr{B}$. The other possibilities for $\left(\mathscr{U}_{1} \vee \mathscr{U}_{2}\right) \cap \mathscr{R} \mathscr{B}$ can be handled similarly. Thus $\left(\mathscr{U}_{1}, \mathscr{V}_{1}\right) \vee\left(\mathscr{U}_{2}, \mathscr{V}_{2}\right) \in \mathscr{F}$ and $\mathscr{F}$ is a sublattice of $\mathscr{L}(\mathscr{C} \mathscr{S}) \times \mathscr{L}(\mathcal{O})$.

Now, for any $\mathscr{U} \in \mathscr{L}(\mathscr{C} \mathscr{S}), \mathscr{V} \in \mathscr{L}(\mathcal{O})$ we have $(\mathscr{U}, \mathscr{U} \cap \mathcal{O}) \in \mathscr{F}$ and $(\mathscr{V} \cap$ $\mathscr{C} \mathscr{S}, \mathscr{V}) \in \mathscr{F}$ so that the natural projections map $\mathscr{F}$ onto $\mathscr{L}(\mathscr{C} \mathscr{S})$ and $\mathscr{L}(\mathcal{O})$, respectively. Thus $\mathscr{F}$ is a subdirect product.

Now define the mapping

$$
\varphi:(\mathscr{U}, \mathscr{V}) \rightarrow \mathscr{U} \vee \mathscr{V} \quad((\mathscr{U}, \mathscr{V}) \in \mathscr{F})
$$

Since $\mathscr{U} \vee \mathscr{V} \subseteq \mathscr{C} \mathscr{S} \vee \mathcal{O}=\mathscr{O}^{+} \cap \mathscr{P O}$, for all $(\mathscr{U}, \mathscr{V}) \in \mathscr{F}$, it follows that $\varphi$ maps $\mathscr{F}$ onto $\mathscr{L}\left(\mathcal{O}^{+} \cap \mathscr{P O}\right)$. In addition, it is easily verified that $\chi$ maps $\mathscr{L}\left(\mathcal{O}^{+} \cap \mathscr{P O}\right)$ into $\mathscr{F}$.

By Proposition $4.1, \varphi$ maps $\mathscr{F}$ onto $\mathscr{L}\left(\mathcal{O}^{+} \cap \mathscr{P O}\right)$. By Corollary 4.6 and Proposition $4.7, \varphi \chi$ is the identity mapping on $\mathscr{F}$ so that, in particular, $\varphi$ is one-to-one. Thus $\varphi$ is a bijection and, therefore, so also is $\chi$ and $\varphi=\chi^{-1}$. Since both mappings are clearly order preserving, they must both be isomorphisms.

Corollary 4.10. The mapping

$$
\psi: \mathscr{V} \rightarrow \mathscr{V} \cap \mathcal{O} \quad\left(\mathscr{V} \in \mathscr{L}\left(\mathcal{O}^{+} \cap \mathscr{P O}\right)\right)
$$

is a retraction of $\mathscr{L}\left(\mathcal{O}^{+} \cap \mathscr{P O}\right)$ onto $\mathscr{L}(\mathcal{O})$.

Proof. Let $\pi$ be the projection of $\mathscr{F}$ onto $\mathscr{L}(\mathcal{O})$. Then $\psi=\chi \pi$ and is therefore a retraction.

\section{Identities for $\mathscr{L}\left(\mathcal{O}^{+} \cap \mathscr{P O}\right)$}

In [20], Rasin obtained a basis of identities for any variety $\mathscr{V}$ in $\mathscr{L}(\mathscr{P O O \mathscr { G }})$ in terms of bases for $\mathscr{V} \cap \mathscr{C} \mathscr{S}$ and $\mathscr{V} \cap \mathscr{B}$. This extended the results of Petrich [10] 
in which a basis of identities was given for any variety $\mathscr{V} \in \mathscr{L}(\mathscr{O B G})$ in terms of bases for $\mathscr{V} \cap \mathscr{G}$ and $\mathscr{V} \cap \mathscr{B}$. The main result of this section represents every variety $\mathscr{V}$ in $\mathscr{L}\left(\mathcal{O}^{+} \cap \mathscr{P O}\right)$ as an intersection of varieties of special form. This is then used to obtain a basis of identities for $\mathscr{V}$ in terms of bases for $\mathscr{V} \cap \mathscr{C} \mathscr{S}$ and $\mathscr{r} \cap \mathcal{O}$.

THEOREM 5.1. If $\mathscr{V} \in \mathscr{L}\left(\mathcal{O}^{+} \cap \mathscr{P O}\right)$, then

$$
\mathscr{V}=(\mathscr{V} \cap \mathscr{C} \mathscr{P})^{*} \cap(\mathscr{V} \cap \mathcal{O})^{+} \cap \mathscr{P O} \text {. }
$$

Proof. Let $\mathscr{W}$ denote the class on the right-hand side of (10). Now $\mathscr{V} \subseteq$ $(\mathscr{V} \cap \mathscr{C} \mathscr{S})^{*}$ and since $\mathscr{V} \subset \mathcal{O}^{+} \cap \mathscr{P O}$, we have $\mathscr{V} \subseteq(\mathscr{V} \cap \mathcal{O})^{+} \cap \mathscr{P O}$. Therefore $\mathscr{r} \subseteq \mathscr{W}$.

Now let $S \in \mathscr{W}$. Since $S \in(\mathscr{V} \cap \mathcal{O})^{+}, S / \mu \in \mathscr{V} \cap \mathcal{O}$. Since $S \in \mathscr{P} \mathcal{O}$, by Lemma 2.6, there exists, for all $e \in E(S)$, an idempotent pure congruence $\rho_{e}$ on $e S e$ such that $e S e / \rho_{e} \in \mathscr{S} \ell \mathscr{G}$. By Lemma $2.5, \rho=V\left\{\rho_{e}^{\#}: e \in E(S)\right\}$ is an idempotent pure congruence on $S$ while $S / \rho \in \mathscr{N} \mathscr{B} \mathscr{G}$. First suppose that $\mathscr{S} \ell \subseteq \mathscr{V}$. Then $\mathscr{S} \ell \subseteq \mathscr{W}$ and

$$
\begin{aligned}
S / \rho & \in \mathscr{W} \cap \mathscr{N} \mathscr{B} \mathscr{G}=[(\mathscr{W} \cap \mathscr{N} \mathscr{B} \mathscr{G}) \cap \mathscr{C} \mathscr{S}] \vee \mathscr{S} \mathscr{l} \\
& =(\mathscr{W} \cap \mathscr{C} \mathscr{S}) \vee \mathscr{S} \ell \\
& \subseteq(\mathscr{V} \cap \mathscr{C} \mathscr{S}) \subseteq \mathscr{S} \ell
\end{aligned}
$$

since $\mathscr{W} \subseteq(\mathscr{V} \cap \mathscr{C} \mathscr{S})^{*}$. Since $\mu \cap \rho=\imath, S$ is a subdirect product of $S / \mu$ and $S / \rho$. Hence

$$
\begin{aligned}
S & \in(\mathscr{V} \cap \mathcal{O}) \vee[(\mathscr{V} \cap \mathscr{C} \mathscr{S}) \vee \mathscr{S} \ell] \\
& =(\mathscr{V} \cap \mathcal{O}) \vee(\mathscr{V} \cap \mathscr{C} \mathscr{S}) \vee \mathscr{S} \ell \\
& \subseteq \mathscr{V}
\end{aligned}
$$

since we are assuming in this case that $\mathscr{S} \ell \subseteq \mathscr{V}$. Thus $\mathscr{W} \subseteq \mathscr{V}$ and equality holds. If $\mathscr{S} \mathscr{\mathscr { V }} \nsubseteq \mathscr{V}$, then $S / \rho \in \mathscr{W} \cap \mathscr{C} \mathscr{S} \subseteq \mathscr{V} \cap \mathscr{C} \mathscr{S}$ so that $S \in(\mathscr{V} \cap \mathcal{O}) \vee(\mathscr{V} \cap \mathscr{C} \mathscr{S})$ $\subseteq \mathscr{V}$ and again $\mathscr{W} \subseteq \mathscr{V}$ so that equality holds in both cases.

Combining Theorems 3.9, 4.4, 5.1 and Lemma 2.14, we obtain

Corollary 5.2. Let $\mathscr{V} \in \mathscr{L}\left(\mathcal{O}^{+} \cap \mathscr{P O}\right)$, let $\mathscr{V} \cap \mathscr{C} \mathscr{S}=\left[u_{\alpha}=v_{\alpha}: \alpha \in A\right]$ and let $\mathscr{V} \cap \mathcal{O}=\left[r_{\beta}=s_{\beta}: \beta \in B\right]$. Then a basis for $\mathscr{V}$ is given by

$$
\begin{aligned}
& u_{\alpha}^{*}=v_{\alpha}^{*} \quad(\alpha \in A) \\
& r_{\beta}^{0}=s_{\beta}^{0} \quad\left(x r_{\beta} y\right)^{0}=\left(x s_{\beta} y\right)^{0} \quad(\beta \in B) \\
& (x a x)^{0}(x b x)^{0}=\left((x a x)^{0}(x b x)^{0}\right)^{0} .
\end{aligned}
$$

Of course, for any variety $\mathscr{V} \subseteq \mathscr{B} \mathscr{G},(\mathscr{V} \cap \mathcal{O})^{+}=(\mathscr{V} \cap \mathscr{B})^{+}$so that Corollary 5.2 can be specialized to $\mathscr{L}(\mathscr{P} O \mathscr{B} \mathscr{G})$. This we now do taking note of the fact (see, 
e.g., Fennemore [2]) that every variety of bands can be defined by means of one identity (in addition to $a^{2}=a$ ). This gives a basis of identities very similar to those given by Rasin [20] in this case.

COROllary 5.3. Let $\mathscr{V} \in[\mathscr{R} \mathscr{B}, \mathscr{P O O B} G]$ and let

$$
\begin{aligned}
\mathscr{V} \cap \mathscr{C} \mathscr{S} & =\left[u_{\alpha}=v_{\alpha}: \alpha \in A\right], \\
\mathscr{V} \cap \mathscr{B} & =\left[r=s, a^{2}=a\right] .
\end{aligned}
$$

Then a basis of identities for $\mathscr{V}$ is

$$
\begin{aligned}
& u_{\alpha}^{*}=v_{\alpha}^{*} \quad(\alpha \in A) \\
& r^{0}=s^{0} \quad(x r y)^{0}=(x s y)^{0},\left(x a^{2} y\right)^{0}=(x a y)^{0} \\
& (x a x)^{0}(x b x)^{0}=\left((x a x)^{0}(x b x)^{0}\right)^{0} .
\end{aligned}
$$

\section{The pseudo operator}

In this section we consider the operator $\mathscr{P}$ in more detail. The behaviour of $\mathscr{P}$ is first determined exactly on $\mathscr{L}(\mathscr{B})$ and then shown to induce an endomorphism on $\mathscr{L}(\mathscr{P O O B} \mathscr{G})$.

Recalling that a monoid is a semigroup with identity, the class $\mathscr{C} \mathscr{R} \mathscr{H}$ of all completely regular monoids is a variety (of algebras with a nullary operation). We denote by $\mathscr{L}(\mathscr{C} \mathscr{R} \mathscr{K})$ the lattice of all subvarieties of $\mathscr{C} \mathscr{R} \mathscr{K}$.

Notation 6.1 ([21], [22]). For any $\mathscr{V} \in \mathscr{L}(\mathscr{C} \mathscr{R})$, let

$$
\text { Mon } \mathscr{V}=\{S \in \mathscr{V}: S \text { is a monoid }\} \text {. }
$$

The next result is essentially covered in Proposition 3.1 and the remarks following Proposition 3.2 of Wismath [22] (see also [21]).

\section{THEOREM 6.2. The mapping}

$$
\text { Mon: } \mathscr{V} \rightarrow \text { Mon } \mathscr{V} \quad(\mathscr{V} \in \mathscr{L}(\mathscr{C} \mathscr{R}))
$$

is a complete lattice homomorphism of $\mathscr{L}(\mathscr{C} \mathscr{R})$ onto $\mathscr{L}(\mathscr{C} \mathscr{R} \mathscr{M})$. Moreover, the congruence on $\mathscr{L}(\mathscr{B})$ induced by Mon is such that each class is finite and the set of all the maximum elements from all the classes is a sublattice of $\mathscr{L}(\mathscr{B})$.

The only part of Theorem 6.2 not covered in [22] is the part concerning the maximum elements. This follows by simple inspection. The lattice $\mathscr{L}(\mathscr{B})$ is presented in Diagram 1, as determined by Fennemore [2], and the elements that are maximum in the classes induced by Mon are circled. 
$\odot$

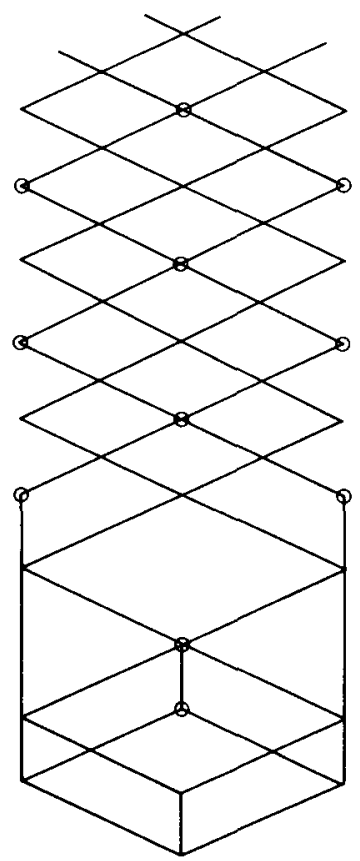

Diagram 1 . The lattice of varieties of bands

LEMMA 6.3. If $\mathscr{V} \in \mathscr{L}(\mathscr{C} \mathscr{R})$, then $\mathscr{P}(\mathscr{V})=\max \{\mathscr{W} \in \mathscr{L}(\mathscr{C} \mathscr{R})$ : Mon $\mathscr{V}=$ Mon $\mathbb{W}$ \}.

Proof. Let $\mathscr{W}$ be the largest variety of completely regular semigroups for which Mon $\mathscr{V}=$ Mon $\mathscr{W}$; this exists by Theorem 6.2. If $S \in \mathscr{W}$ and $e^{2}=e \in S$, then, $e S e \in$ Mon $\mathscr{W}=$ Mon $\mathscr{V} \subseteq \mathscr{V}$ so that $S \in \mathscr{P}(\mathscr{V})$ and $\mathscr{W} \subseteq \mathscr{P}(\mathscr{V})$. On the other hand, if $S \in \operatorname{Mon}(\mathscr{P}(\mathscr{V}))$, then $S \in \mathscr{V}$ and therefore $S \in \operatorname{Mon}(\mathscr{V})$. But Mon $\mathscr{V}$ is clearly contained in $\operatorname{Mon}(\mathscr{P}(\mathscr{V}))$ so that $\operatorname{Mon}(\mathscr{P}(\mathscr{V}))=\operatorname{Mon} \mathscr{V}$. It follows from the definition of $\mathscr{W}$ that $\mathscr{P}(\mathscr{V}) \subseteq \mathscr{W}$ and equality holds.

THEOREM 6.4. The operator $\mathscr{P}$ is an endomorphism on $\mathscr{L}(\mathscr{B})$.

Proof. This follows easily from Lemma 6.3 and Theorem 6.2. 
LEMMA 6.5. For $\mathscr{W} \in \mathscr{L}(\mathscr{P O O B G})$

$$
\mathscr{P}(\mathscr{W})=\mathscr{P}(\mathscr{W} \cap \mathscr{B}) \vee \mathscr{P}(\mathscr{W} \cap \mathscr{C} \mathscr{S})
$$

Proof. We have $\mathscr{P}(\mathscr{W}) \in \mathscr{L}(\mathscr{P O O B G})$ by (3), so that

$$
\begin{aligned}
\mathscr{P}(\mathscr{W}) & =[\mathscr{P}(\mathscr{W}) \cap \mathscr{B}] \vee[\mathscr{P}(\mathscr{W}) \cap \mathscr{C} \mathscr{S}] \quad \text { (by Proposition } 4.1(\mathrm{ii})) \\
& =\mathscr{P}(\mathscr{W} \cap \mathscr{B}) \vee \mathscr{P}(\mathscr{W} \cap \mathscr{C} \mathscr{P}) .
\end{aligned}
$$

PROPOSITION 6.6. $\mathscr{P}$ induces a retraction of $\mathscr{L}(\mathscr{P O O B} \mathscr{G})$.

Proof. Let $\mathscr{U}, \mathscr{V} \in \mathscr{L}(\mathscr{P O O B G})$. Then

$$
\begin{aligned}
\mathscr{P}(\mathscr{U} \vee \mathscr{V})= & \mathscr{P}([\mathscr{U} \vee \mathscr{V}] \cap \mathscr{B}) \vee \mathscr{P}([\mathscr{U} \vee \mathscr{V}] \cap \mathscr{C} \mathscr{P})(\text { by Lemma 6.5) } \\
= & \mathscr{P}([\mathscr{U} \cap \mathscr{B}] \vee[\mathscr{V} \cap \mathscr{B}]) \vee \mathscr{P}([\mathscr{U} \cap \mathscr{C} \mathscr{S}] \vee[\mathscr{V} \cap \mathscr{C} \mathscr{S}]) \\
& \quad \text { (by Corollary 4.5) } \\
= & \mathscr{P}(\mathscr{U} \cap \mathscr{B}) \vee \mathscr{P}(\mathscr{V} \cap \mathscr{B}) \vee \mathscr{P}(\mathscr{U} \cap \mathscr{C} \mathscr{P}) \vee \mathscr{P}(\mathscr{V} \cap \mathscr{C} \mathscr{S}) \\
& \quad(\text { by Lemma } 2.13 \text { and Theorem 6.4) } \\
= & \mathscr{P}(\mathscr{U} \cap \mathscr{B}) \vee \mathscr{P}(\mathscr{U} \cap \mathscr{C} \mathscr{P}) \vee \mathscr{P}(\mathscr{V} \cap \mathscr{B}) \vee \mathscr{P}(\mathscr{V} \cap \mathscr{C} \mathscr{S}) \\
= & \mathscr{P}(\mathscr{U}) \vee \mathscr{P}(\mathscr{V}) \quad(\text { by Lemma 6.5). }
\end{aligned}
$$

The result now follows from (3).

\section{Relationships between $\mathscr{P},(-)^{+}$and $(-)^{p}$}

In this section we introduce a new operator $(-)^{p}$ and obtain some general relationships between the operators $\mathscr{P},(-)^{+}$and $(-)^{p}$. We require some preliminary observations.

LEMMA 7.1. Let $S$ be a completely regular semigroup, $e^{2}=e \in S$ and $\rho$ be $a$ congruence on eSe. If $\rho \subseteq \mathscr{H}$ then $\rho^{\#} \subseteq \mathscr{H}$.

Proof. Let $e$ and $S$ be as in the statement of the lemma. The claim of the lemma is clearly equivalent to the claim that $\left(\mu_{e S e}\right)^{\#} \subseteq \mu$ and so to the assertion that $\mu_{l e S e}=\mu_{e S e}$. But this is true in any regular semigroup by [5, Corollary 6], and so the result follows.

Recall from Section 2 that a congruence $\rho$ on a completely regular semigroup $S$ is said to be idempotent pure if $(e, a) \in \rho$ and $e^{2}=e$ imply that $a^{2}=a$. This 
terminology is consistent with the terminology of [13]. For a completely regular semigroup $S$ and any congruence $\rho$ on $S$, it is clear that $\rho$ is idempotent pure if and only if $\rho \cap \mathscr{H}=\imath$.

Notation 7.2. For any $\mathscr{V} \in \mathscr{L}(\mathscr{C} \mathscr{R})$, let

$\mathscr{V}^{p}=\{S \in \mathscr{C} \mathscr{R}: S / \rho \in \mathscr{V}$ for some idempotent pure congruence $\rho$ on $S\}$.

In general, $\mathscr{V}^{p}$ may not be a variety. However, in some important cases this will be so (see Corollary 7.4).

PROPOSITION 7.3. For any class $\mathscr{X}$ of completely regular semigroups (i) $\mathscr{P}\left(\mathscr{K}^{+}\right)=$ $(\mathscr{P} \mathscr{K})^{+}$and (ii) $\mathscr{P}\left(\mathscr{X}^{p}\right)=(\mathscr{P} \mathscr{X})^{p}$.

Proof. Let $S \in \mathscr{P}\left(\mathscr{K}^{+}\right)$. Then, for all $e \in E(S)$, there exists a congruence $\rho_{e}$ on $e S e$ such that $\rho_{e} \subseteq \mathscr{H}$ and $e S e / \rho_{e} \in \mathscr{K}$. By Lemmas 7.1 and $2.5, \rho=$ $\vee\left\{\rho_{e}^{\#}: e \in E(S)\right\} \subseteq \mathscr{H}$ and $S / \rho \in \mathscr{P}(\mathscr{K})$. Thus $S \in(\mathscr{P} \mathscr{K})^{+}$and $\mathscr{P}\left(\mathscr{K}^{+}\right) \subseteq$ $(\mathscr{P} \mathscr{K})^{+}$. Now let $S \in(\mathscr{P} \mathscr{K})^{+}$and $\rho$ be a congruence on $S$ with $\rho \subseteq \mathscr{H}$ and $S / \rho \in \mathscr{P} \mathscr{K}$. For all $e \in E(S)$, let $\rho_{e}=\rho \mid e S e$. then $\rho_{e} \subseteq \mathscr{H}$ and $e S e / \rho_{e} \in \mathscr{K}$. Thus $e S e \in \mathscr{K}^{+}$and $S \in \mathscr{P}\left(\mathscr{X}^{+}\right)$so that $(\mathscr{P} \mathscr{K})^{+} \subseteq \mathscr{P}\left(\mathscr{X}^{+}\right)$, as required.

The proof of part (ii) is very similar to that of (i).

COROLlaRY 7.4. (i) $(\mathscr{S} \ell)^{p}=\mathscr{B}$. (ii) $(\mathscr{S} \ell \mathscr{G})^{p}=\mathscr{O}$ (iii) $(\mathscr{N} \mathscr{B} \mathscr{G})^{p}=\mathscr{P O}$.

Proof. (i) and (ii) are clear. For (iii) we have, by Proposition 7.3, $(\mathscr{N} \mathscr{B} \mathscr{G})^{p}=$ $(\mathscr{P} \mathscr{S} \ell \mathscr{G})^{p}=\mathscr{P}\left(\mathscr{S} \ell \mathscr{G}^{p}\right)=\mathscr{P} \mathcal{O}$. Note that, for any $\mathscr{U} \in \mathscr{L}(\mathscr{B})$, we have $\mathscr{P}(\mathscr{U}) \subseteq$ $\mathscr{B} \subseteq(\mathscr{T})^{p} \subseteq(\mathscr{U} \mathscr{B} \mathscr{G})^{p}$ so that $\mathscr{P}(\mathscr{L}) \vee \mathscr{L} \mathscr{B} \mathscr{G} \subseteq(\mathscr{L} \mathscr{B} \mathscr{G})^{p}$.

The next theorem and its corollaries indicate that certain relationships that hold when the operators $\mathscr{P},(-)^{+},(-)^{p}$ are applied to $\mathcal{O}$ in fact hold more generally.

TheOREM 7.5. Let $\mathscr{Q} \in \mathscr{L}(\mathscr{B}), \mathscr{K} \in \mathscr{L}(\mathscr{C} \mathscr{R})$ be such that

$$
\mathscr{P} \mathscr{U} \vee \mathscr{U} \mathscr{B} \mathscr{G} \subseteq \mathscr{K} \subseteq(\mathscr{U} \mathscr{B} \mathscr{G})^{p} .
$$

Then $\mathscr{X}^{+} \cap \mathscr{P} \mathscr{X}=\mathscr{K} \vee \mathscr{P} \mathscr{Q} \mathscr{B} \mathscr{G}$.

Proof. Clearly $\mathscr{X} \subseteq \mathscr{X}^{+} \cap \mathscr{P P}$ and $\mathscr{P} \mathscr{L} \mathscr{B} \mathscr{G} \subseteq \mathscr{P} \mathscr{X}$ by (11). Let $S \in \mathscr{P P} \mathscr{U} \mathscr{B} \mathscr{G}$. Then $\mathscr{H}$ is a congruence on $S$ and $S / \mathscr{H} \in \mathscr{B} \cap \mathscr{P} \mathscr{Q} \mathscr{B} \mathscr{G}=\mathscr{P} \mathscr{U} \subseteq \mathscr{K}$ so that $S \in \mathscr{X}^{+}$. Therefore $\mathscr{X} \cup \mathscr{P} \mathscr{U} \mathscr{B} \mathscr{G} \subseteq \mathscr{X}^{+} \cap \mathscr{P} \mathscr{X}$ and $\mathscr{K} \vee \mathscr{P} \mathscr{L} \mathscr{B} \mathscr{G} \subseteq \mathscr{K}^{+} \cap \mathscr{P} \mathscr{K}$. Now let $S \in \mathscr{X}^{+} \cap \mathscr{P} \mathscr{X}$. Then $S / \mu \in \mathscr{X}$. Also, for all $e \in E_{S}$, there exists, by (11) an idempotent pure congruence $\rho_{e}$ on $e S e$ such that $e S e / \rho_{e} \in \mathscr{U} \mathscr{B} \mathscr{G}$. By 
Lemma 2.5, $\rho_{\mathrm{e}}^{\#}$ is idempotent pure and also $\rho=V\left\{\rho_{e}^{\#}: e \in E(S)\right\}$ so that $S / \rho \in \mathscr{P} \mathscr{U} \mathscr{B} \mathscr{G}$. Since $\rho \cap \mu=\iota, S \subseteq S / \mu \times S / \rho \in \mathscr{X} \vee \mathscr{P} \mathscr{U} \mathscr{B} \mathscr{G}$. Thus $\mathscr{X}^{+} \cap$ $\mathscr{P} \mathscr{K} \subseteq \mathscr{K} \vee \mathscr{P} \mathscr{U} \mathscr{B} \mathscr{G}$ and equality follows.

Theorem 7.5 has some interesting special cases. In applying the theorem, it is necessary to choose a pair of varieties $(\mathscr{U}, \mathscr{X})$ with $\mathscr{U} \in \mathscr{L}(\mathscr{B})$ and satisfying (11). Note that if $\mathscr{U}=\mathscr{S} \ell$, then $\mathscr{P} \mathscr{U}=\mathscr{N} \mathscr{B}, \mathscr{P} \mathscr{U} \vee \mathscr{U} \mathscr{B} \mathscr{G}=\mathscr{N} \mathscr{B} \vee \mathscr{S} \mathscr{G}=$ $\mathcal{O N} \mathscr{B} \mathscr{G}$ and $(\mathscr{S} \ell \mathscr{G})^{p}=0$. Thus (11) becomes $\mathcal{O} \mathcal{N} \mathscr{B} \mathscr{G} \subseteq \mathscr{K} \subseteq \mathcal{O}$ and we have

COROllary 7.6. Let $\mathscr{K} \in \mathscr{L}(\mathscr{C} \mathscr{R})$. If $\mathcal{O} \mathscr{N} \mathscr{B} \mathscr{G} \subseteq \mathscr{K} \subseteq \mathcal{O}$, then

$$
\mathscr{K}^{+} \cap \mathscr{P} \mathscr{K}=\mathscr{K} \vee \mathscr{N} \mathscr{B} \mathscr{G}
$$

while if $\mathscr{N} \mathscr{B} \mathscr{G} \subseteq \mathscr{K} \subseteq \mathscr{P O}$, then $\mathscr{X}=\mathscr{X}^{+} \cap \mathscr{P} \mathscr{X}$.

Proof. These results follow by applying Theorem 7.5 to the pairs $(\mathscr{P} \ell, \mathscr{K})$ and $(\mathscr{N} \mathscr{B}, \mathscr{K})$, respectively, and invoking Corollary 7.4.

EXAMPLE 1. Let $(\mathscr{U}, \mathscr{K})=(\mathscr{S}, \mathscr{O} \mathscr{B} \mathscr{G})$. Then $\mathscr{K}^{+}=\mathscr{O} \mathscr{B} \mathscr{G}^{+}=\mathscr{B} \mathscr{G}$ and $\mathscr{P} \mathscr{Q} \mathscr{B} \mathscr{G}$ $=\mathscr{N} \mathscr{B} \mathscr{G}$ so that from (16) we have $\mathscr{B} \mathscr{G} \cap \mathscr{P} O \mathscr{B} \mathscr{G}=\mathscr{O} \mathscr{B} \mathscr{G} \vee \mathscr{N} \mathscr{B} \mathscr{G}$ or $\mathscr{P} O \mathscr{B} \mathscr{G}=$ $\mathcal{O B G} \vee \mathscr{N} \mathscr{B} \mathscr{G}$ which is Corollary 5.4 of [6].

ExAMPle 2. Let $(\mathscr{U}, \mathscr{K})=(\mathscr{S} \ell, \mathcal{O})$. Then (12) yields $\mathscr{O}^{+} \cap \mathscr{P} \mathscr{O}=\mathscr{O} \vee \mathscr{N} \mathscr{B} \mathscr{G}$ which, recalling that $\mathcal{O}^{+}=I$, we see is Theorem 5.3 of [6].

\section{References}

[1] A. P. Birjukov, 'Varieties of idempotent semigroups', Algebra i Logika 9 (1970), 255-273 (Russian)

[2] C. F. Fennemore, 'All varieties of bands', Math. Nachr. 4 (1971); I: 237-255; II: 253-262.

[3] J. A. Gerhard, 'The lattice of equational classes of idempotent semigroups', J. Algebra 15 (1970), 195-224.

[4] J. A. Gerhard, and M. Petrich, 'All varieties of regular orthogroups', manuscript.

[5] T. E. Hall, 'On regular semigroups', J. Algebra 24 (1973), 1-24.

[6] T. E. Hall, and P. R. Jones, 'On the lattice of varieties of bands of groups', Pacific J. Math. 91 (1980) 327-337.

[7] J. M. Howie, An introduction to semigroup theory (Academic Press, London, 1976).

[8] P. R. Jones, 'Completely simple semigroups: free products, free semigroups and varieties', Proc. Roy. Soc. Edinburgh Sect. A 88 (1981), 293-313.

[9] P. R. Jones, 'Varieties of completely regular semigroups', J. A ustral. Math. Soc. (Series A) 35 (1983), 227-235.

[10] M. Petrich, 'Varieties of orthodox bands of groups', Pacific J. Math. 58 (1975), 209-217.

[11] M. Petrich, 'Certain varieties and quasivarieties of completely regular semigroups', Canad. $J$. Math. 29, (1977), 1171-1197.

[12] M. Petrich, 'On the varieties of completely regular semigroups', Semigroup Forum 25 (1982) 153-169. 
[13] M. Petrich, and N. R. Reilly, 'A network of congruences on an inverse semigroup', Trans. Amer. Math. Soc. 270 (1982), 309-325.

[14] M. Petrich and N. R. Reilly, 'Bands of groups with universal properties', Monatshefte für Mathematik 94 (1982), 45-67.

[15] M. Petrich and N. R. Reilly, 'Varieties of groups and of completely simple semigroups', Bull. Austral. Math. Soc. 23 (1981), 339-359.

[16] M. Petrich and N. R. Reilly, 'Near varieties of idempotent generated completely simple semigroups', Algebra Universalis 16 (1983), 83-104.

[17] M. Petrich and N. R. Reilly, 'All varieties of central completely simple semigroups', Trans. Amer. Math. Soc. 280 (1983), 623-636.

[18] M. Petrich and N. R. Reilly, 'Certain homomorphism of the lattice of varieties of completely simple semigroups', J. A ustral. Math. Soc. (to appear).

[19] V. V. Rasin, 'On the lattice of varieties of completely simple semigroups', Semigroup Forum 17 (1979), 113-122.

[20] V. V. Rasin, 'On the varieties of Cliffordian semigroups', Semigroup Forum 23 (1981), 201-220.

[21] S. Wismath, 'The lattice of varieties and pseudovarieties of band monoids', Thesis, Simon Fraser University, 1983.

[22] S. Wismath, 'The lattice of varieties and pseudovarieties of band monoids', manuscript.

Department of Mathematics and Statistics

Simon Fraser University

Burnaby, B. C. V5A 1S6

Canada 